\title{
Particle physics measurements at the highest energies with the Pierre Auger Observatory
}

\author{
Sofia ANDRINGA ${ }^{* 1}$, for the Pierre Auger Collaboration ${ }^{2}$ \\ ${ }^{1}$ LIP - Lab. Instrum. Física Exp. Partículas \\ ${ }^{2}$ Observatorio Pierre Auger, Av. San Martin Norte 304, 5613 Malargüe, Argentina \\ full author list: www.auger.org/archive/authors_2013_06.html \\ E-mail: sofia.andringa.diasecern.ch
}

\begin{abstract}
The Pierre Auger Observatory measures cosmic rays with energies between $10^{17.5} \mathrm{eV}$ and $10^{20} \mathrm{eV}$, based on air shower sampling at ground, complemented with shower development measurements with a smaller duty-cycle. The cross-section for the primary interaction of $10^{18} \mathrm{eV}$ protons with air has been measured by analysing the maximum of shower development in the atmosphere. This corresponds to a centre-of-mass energy of $57 \mathrm{TeV}$, and the LHC results show the same evolution of the proton-proton cross-section at intermediate energies. The depth of shower maximum is sensitive to cross-section and primary mass. Its energy evolution indicates a change towards the behavior expected for heavier primaries or larger cross-sections. We will show also the results on other observables related to primary nuclear mass composition.
\end{abstract}

The European Physical Society Conference on High Energy Physics

18-24 July, 2013

Stockholm, Sweden

\footnotetext{
* Speaker.
} 


\section{The Pierre Auger Observatory and the Highest Energy Beams}

The Pierre Auger Observatory is a hybrid detector in which cosmic ray showers are sampled with a ground array of $3000 \mathrm{~km}^{2}$ and imaged by surrounding fluorescence telescopes, allowing for direct observation of the shower development in a subset of the data sample. Details can be found in [1]. The observatory aims at exploring the highest available energy beams for particle physics studies and to study their astrophysical origins.

Different nuclei, photons and neutrino primaries can be identified through the characteristics of the showers they initiate in the atmosphere. Heavy nuclei, with a high interaction cross-section, will initiate showers high up in the atmosphere and, with a high multiplicity, the showers will develop faster and will produce a larger number of muons than a proton initiated shower. Photons will interact later and nearly all their energy will be transferred to a similar electromagnetic cascade but with fewer muons. Neutrinos are the only primaries that can transverse all of the atmosphere horizontally and interact only close to the Observatory, so they can be distinguished from other horizontal showers, in which the electromagnetic component has been absorbed and only muons are left. Neutrons and protons will be indistinguishable, except for the fact that, as other neutral particles, the arrival directions of neutrons can be related to their source positions, even at low energies.

The collaboration has conducted dedicated searches for all neutral primaries but no positive indications were found so far. Limits on neutrons, photons and neutrinos are described in [2, 3, 4], respectively. Both photons and neutrinos are expected to be created in the interaction of hadronic primaries with the CMB. The corresponding fluxes at Earth are expected to be small, but the sensitivity of Auger will soon reach the upper bound of the predictions for primary protons. Other production mechanisms are already severely constrained by the present limits. We concentrate here on the exploration of the charged nuclei beams.

\section{FD measurements of $X_{\max }$ : proton-air interaction cross-section and nuclear beam composition}

One of the main variables to distinguish primary nuclei is the atmospheric depth at which the shower reaches the maximum number of particles, $X_{\max }=X_{1}+\Delta X$, determined by the point of first interaction $\left(X_{1}\right)$ and the slant depth needed for the shower to develop up to its maximum $(\Delta X)$. Both components are smaller, and have less fluctuations, for showers initiated by heavy primaries, with a large interaction cross-section and the energy subdivided between several nucleons. An exponential tail at high $X_{\max }$, visible in figure 1, is dominated by the primaries with the deepest $X_{1}$.

In [5], an unbiased sample of high $X_{\max }$ events between 1 and $3 \mathrm{EeV}$ is selected to measure the cross-section of inelastic proton-air collisions. The proton-proton inelastic cross-section at $\sqrt{s}=57 \pm 6 \mathrm{TeV}$ is obtained using the Glauber formalism: the value of $92 \pm 7$ (stat) ${ }_{-11}^{+9}$ (syst) \pm 7 (Glauber) $\mathrm{mb}$ is in good agreement with the extrapolation from the LHC measurements by almost one order of magnitude in energy. The main systematics uncertainties arise from the modeling of hadronic interactions, namely their elasticity, and possible beam contamination by photon and Helium primaries. While direct photon limits constrain the upward errors, for Helium a $25 \%$ contamination is assumed for reference and leads to a higher downward error. 

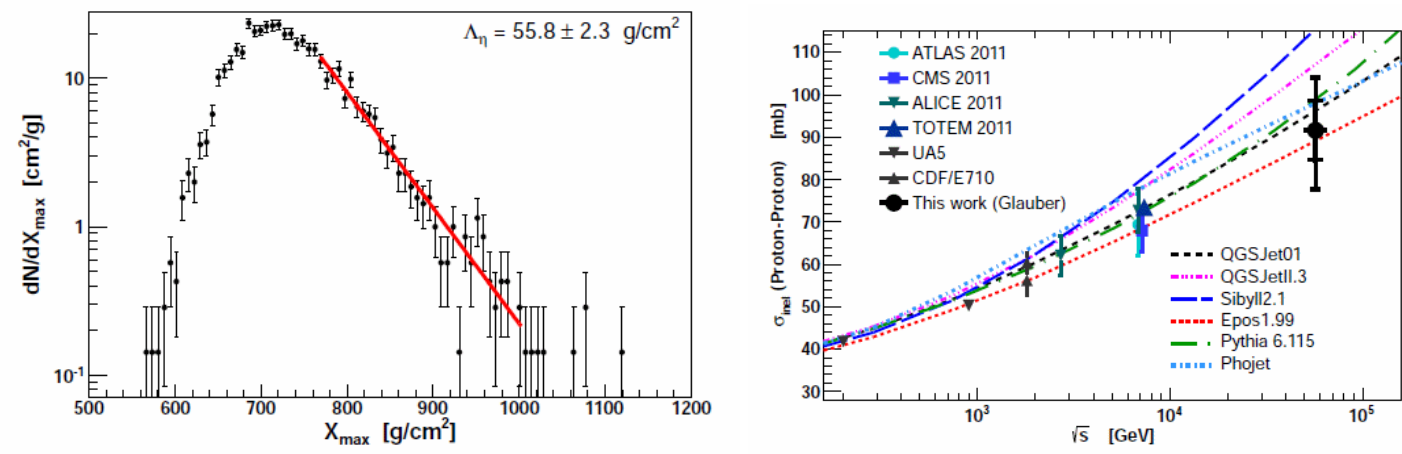

Figure 1: The left plot shows the exponential fit to the tail of the $X_{\max }$ distribution; the right plot shows the derived proton-proton inelastic cross-section [5].

Measurements at still higher energy could also be possible in Auger, as long as the relative presence of Helium can be assumed to be limited. At present, the evolution of the first two moments of the $X_{\max }$ distributions are used to extract information about the nuclear mass composition of the samples.

As shown in [7], $\left\langle X_{\max }\right\rangle$ depends linearly on the average of the logarithm of the nuclear mass $\langle\ln A\rangle$, while its dispersion depends both on the $\langle\ln A\rangle$ and $\sigma^{2}(\ln A)$. A conversion relation between the moments of $X_{\max }$ and $\ln A$ can be obtained in the framework of a given hadronic interaction model. Fig 2 shows how the present Auger results indicate that while at low energies the data is compatible with a proton dominated beam, the average nuclear mass increases with energy, with increasingly smaller dispersions. Such a scenario would have strong implications to astrophysics source modeling, but this interpretation depends on the validity of the hadronic interaction modeling at high energy. Naturally, the mixture between nuclei within hydrogen and iron defines a possible region in the $\langle\ln A\rangle . v s . \sigma^{2}(\ln A)$ plane even without strong assumptions on the sources. Given lower systematic uncertainties, the data can be used in the future to constrain the hadronic interaction model properties and the extrapolation of low energy laboratory measurements to energies beyond the LHC.

\section{SD measurements of muons:}

\section{total number of muons and muonic shower development}

The FD data is limited in statistics and the SD can be used to extend the available beam energies even further, giving also independent measurements in the overlap region. Of special importance are the measurements based on muons, more closely related to the hadronic shower core and undetected by the FD. In the Pierre Auger Observatory, ground measurements are made by Water Cherenkov Detectors, sensitive to a combination of electromagnetic and muonic signals.

The fraction of muon signal at a $1000 \mathrm{~m}$ from the core ${ }^{1}$ varies as a function of zenith angle. For $10 \mathrm{EeV}$ showers, it goes from around $50 \%$ at the median angle of $38^{\circ}$ up to $90 \%$ for very inclined showers, of $60^{\circ}$. This evolution has been consistently obtained by two different methods [8] which

\footnotetext{
${ }^{1} 1000 \mathrm{~m}$ is the reference distance at which the energy estimator is defined.
} 

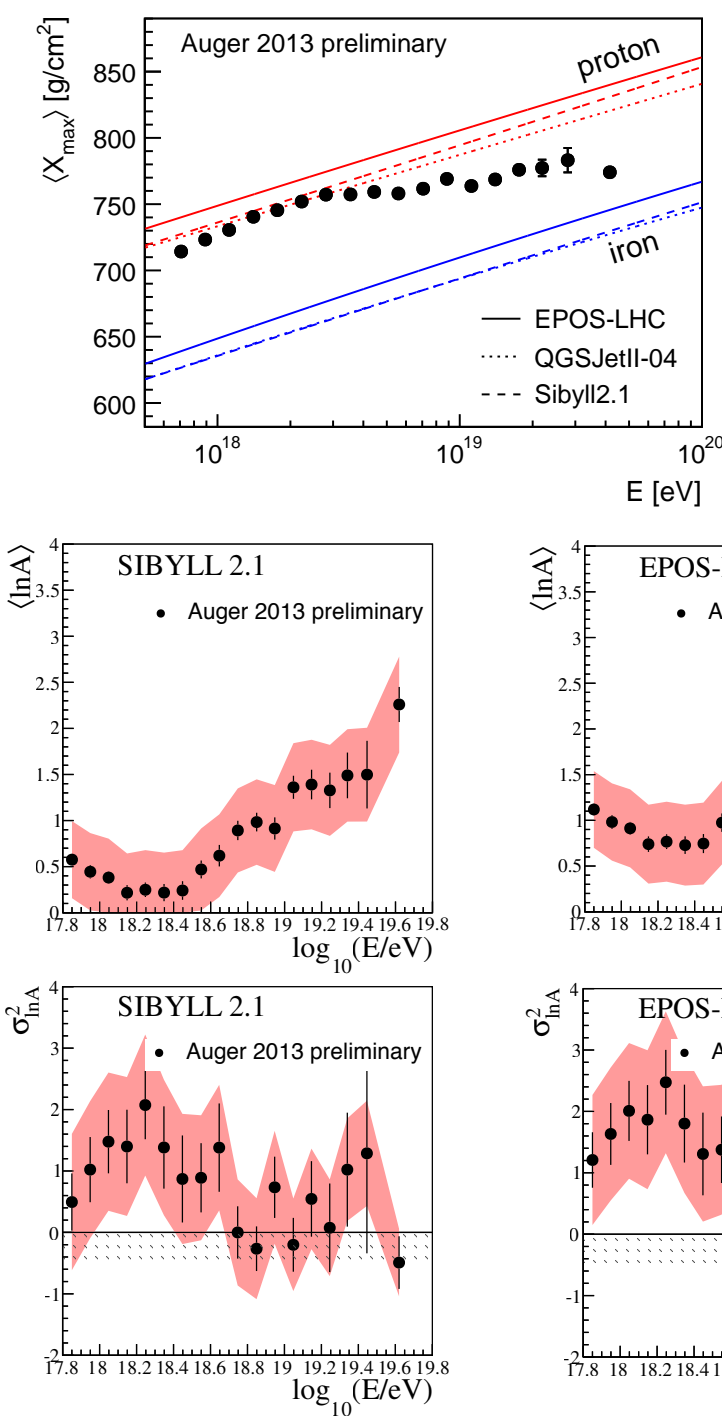
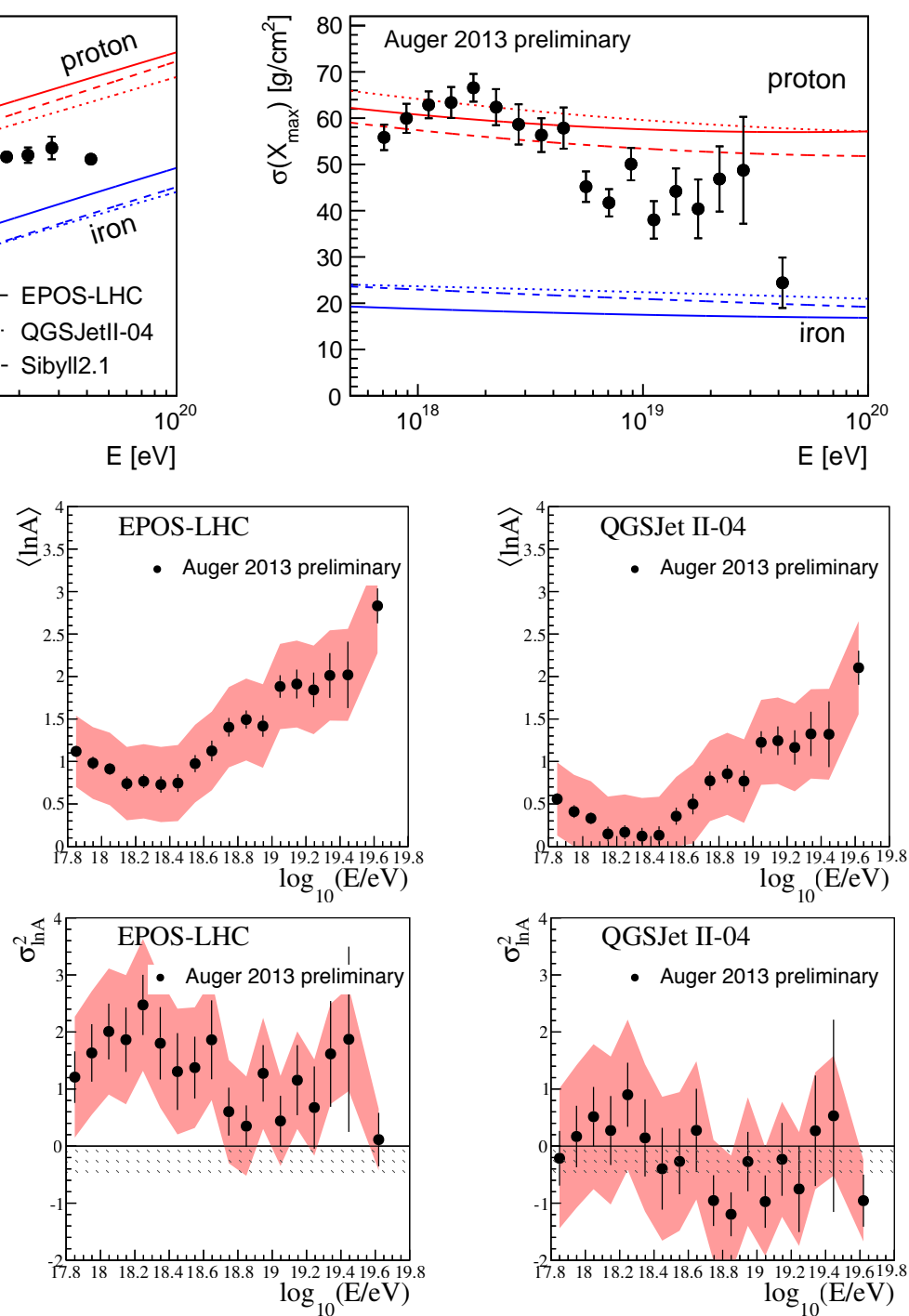

Figure 2: The top plots show the evolution of the first moments of the $X_{\max }$ distribution with energy; the lower plots show how they can be interpreted in terms of the logarithm of primary mass, within each hadronic interaction model $[6,7]$.

explore the fact that muons leave a large signal concentrated in time, while the electromagnetic component of the shower is smoother and spread in time. The ratio of the muon measurement to the expectation for proton primaries is constant within uncertainties, but at a value $40 \%$ higher, similar to the expectation for iron primaries. An independent method [9], using events above $62^{\circ}$, finds that the disagreement with the predictions increases with energy, from $\log (\mathrm{E} / \mathrm{eV})=18.5$ to 19.5, pointing to a higher deficit of muons in the models at higher energies. These results, shown in figure 3 , seem to be incompatible with the average mass results obtained from the electromagnetic shower in the overlaping energy region. The quantification of this incompatibility and of the slopes of the evolution of the number of muons with zenith angle and energy is still limited by the systematic uncertainties in the energy scale and energy calibration of the experiment, even if they 

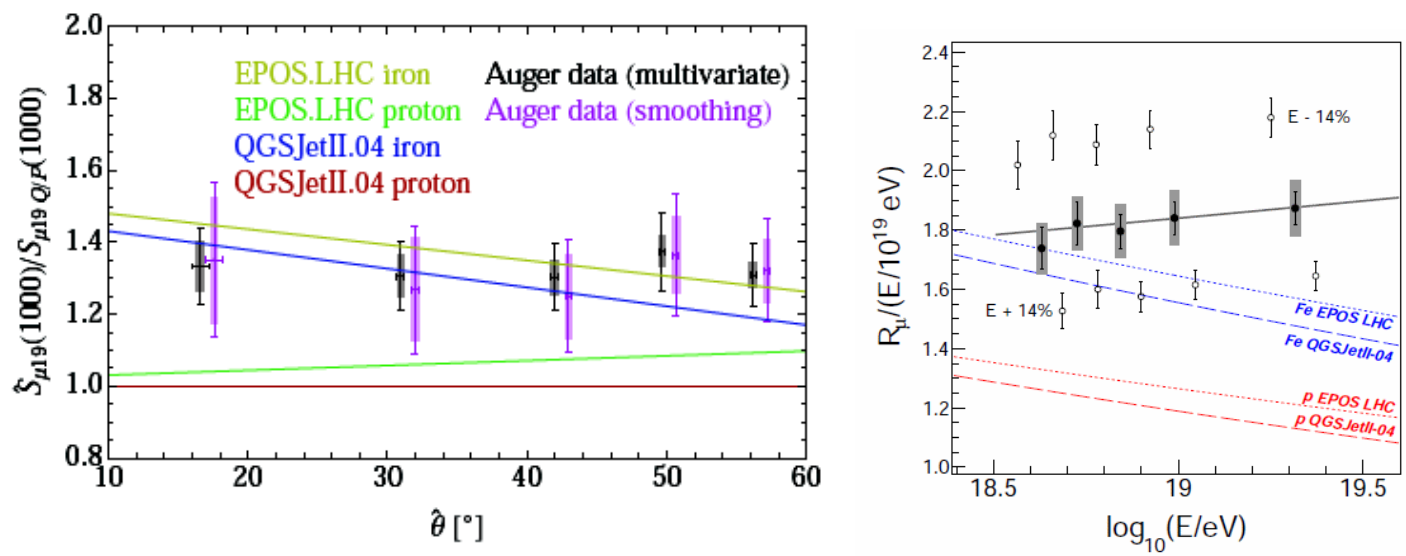

Figure 3: The left plot shows the evolution of the number of muons with zenith angle, as measured by different methods in relation to the expectations of protons simulated with QGSJetII-04 at $10 \mathrm{EeV}$; the right plot shows the evolution with energy for the number of muons in inclined events at zenith angle of 60 degrees $[8,9]$.
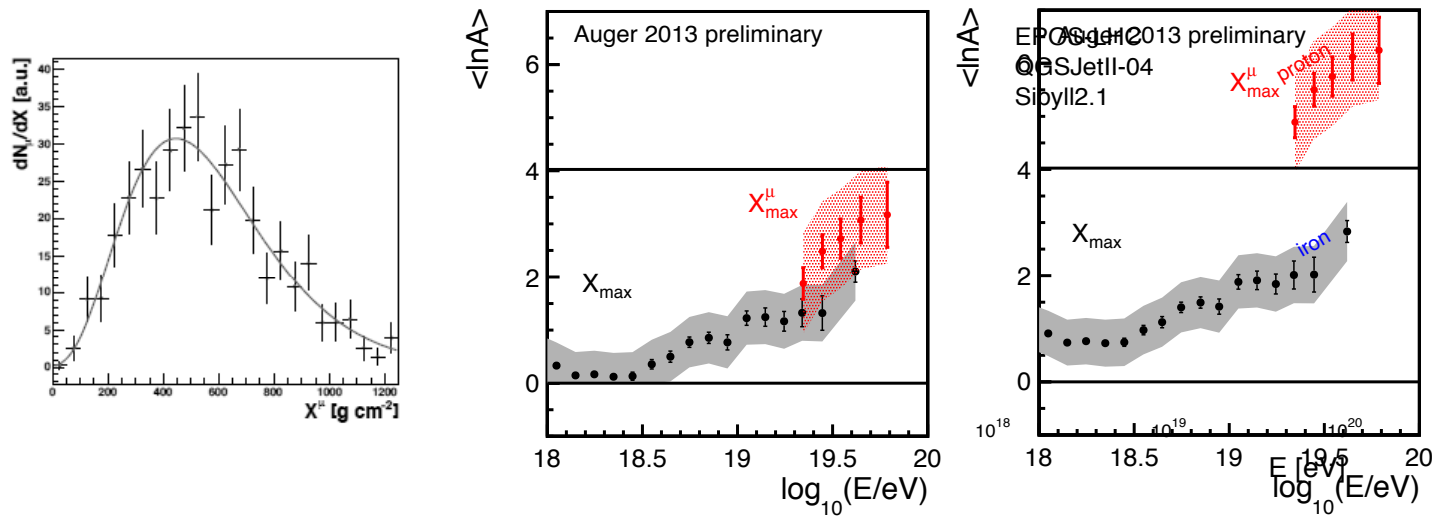

Figure 4: The left plot shows the longitudinal profile of muon production in an event with $E=(92 \pm 3) \mathrm{EeV}$, with an associated fit to extract $X_{\max }^{\mu}$; the next panels show the $\langle\ln A\rangle$ as extracted from $\left\langle X_{\max }\right\rangle$ and $\left\langle X_{\max }^{\mu}\right\rangle$ for QGSJetII-04 (middle) and EPOS-LHC (right), respectively from ref. [12].

were significantely improved recently [10].

The compatibility between electromagnetic and hadronic shower description can be further checked by the analysis of the longitudinal development of the muonic component. The Pierre Auger Collaboration uses muon arrival times at each SD station to construct a muon production longitudinal profile, similar to the electromagnetic profile observed in the FD, and measure the position of its maximum, $X_{\max }^{\mu}$, for each event. In fact, muons travel in nearly straight lines at approximately the velocity of light from their production point near the shower axis, and their delay relative to the shower front can be converted to a production point given they are identified at a sufficiently large distance from the shower core. The resulting longitudinal profile is shown in figure 4 (left).

The analysis in [11] uses events at $60^{\circ}$ and $\log (E / e V)>19.1$ to construct a new "elongation rate" of the showers. The total number of muons is not used directly, though high numbers al- 
low a better reconstruction of the profile of each event. A systematic uncertainty of $17 \mathrm{~g} / \mathrm{cm}^{2}$ is dominated by differences in the shower shapes expected in different hadronic interaction models. Notice that $X_{\max }^{\mu}$ shares with $X_{\max }$ the information on $X_{1}$ or the primary cross-section, but is differently sensitive to $\Delta X$, as the maximum production of muons is reached before the maximum number of electromagnetic particles. $X_{\max }-X_{\max }^{\mu}$ tends to be fairly constant irrespective of the primary particle considered, but the expected value depends on the details of the shower development implemented in each hadronic interaction model.

As for $\left\langle X_{\max }\right\rangle$, also $\left\langle X_{\max }^{\mu}\right\rangle$ can be converted to $\langle\ln A\rangle$, in the context of each hadronic interaction model, giving a strong direct test of each implementation, as shown in figure 4. Independent of the primary interaction cross-section, this difference tests other particle physics variables like the multiplicity, elasticity or the baryon to meson ratio, and the consistency of the shower description. As an example, we have checked that in the present implementation of EPOS-LHC there is an inconsistency that did not exist before, and which is thought to be due to the tuning of the model to reproduce the rapidity gap distributions measured by ATLAS at the LHC [13].

\section{Summary and outlook}

The Pierre Auger Observatory aims at using the highest energy beams to study particle physics, as well as to study their astrophysical origin. The $X_{\max }$ measurements are compatible with a proton dominated flux at the EeV scale allowing for a measurement of the proton-air interaction crosssection, which can be converted to proton-proton inelastic cross-section at a centre-of-mass energy of around $60 \mathrm{TeV}$, consistent with the extrapolation from the LHC measurements at $7 \mathrm{TeV}$. This is the first measurement at the Pierre Auger Observatory of a direct particle physics parameter, and sets a good calibration point for future analyses.

At higher energies, the nuclear composition seems to be more complex. The analysis of the same basic variable, the depth of electromagnetic shower maximum, in terms of the logarithm of nuclear mass indicates that this increases with energy, but with decreasing fluctuations. This behaviour can in the future be used to constrain the hadronic interaction models that favor unphysical interpretations of the data.

Hadronic interaction model testing at very high energy is mostly limited by systematic uncertainties on the energy scale and calibration of the detector, which has recently been improved. New measurements on the number of muons at ground indicate that hadronic models tend to predict less muons than observed in data, compatible with a even heavier composition than that indicated by the electromagnetic shower development data.

Auger has developed a new method to test the hadronic shower development by constructing the longitudinal profile of muon production. This new muonic shower maximum extends the electromagnetic shower maximum analysis to higher energies with a similar trend, approaching the expectations for heavier showers as the energy increases. The difference of the electromagnetic and muonic shower maxima - reflected in their different interpretation in terms of $\langle\ln A\rangle$ - is insensitive to the first interaction point and thus less sensitive to the cross-section and instead can be used to test the other parameters relevant for high energy shower development, opening the way to new measurements in the future. 


\section{References}

[1] The Pierre Auger Collaboration, Nucl. Instrum. Methods A 523, 50 (2004); Nucl. Instrum. Methods A 613, 29 (2010). Nucl. Instrum. Methods A 620, 227 (2010).

[2] The Pierre Auger Collaboration, "A Search for Point Sources of EeV Neutrons”, ApJ, 760 (2012) 148;

[3] The Pierre Auger Collaboration, "Upper limit of the cosmic-ray photon fraction at EeV energies from the Pierre Auger Observatory”, Astroparticle Physics 31 (2009) 399-406;

[4] The Pierre Auger Collaboration, "Ultra-High Energy Neutrinos at the Pierre Auger Observatory", Advances in High Energy Physics, 2013 (2013) 708680;

[5] The Pierre Auger Collaboration, "Measurement of the proton-air cross-section at sqrt(s)=57 TeV with the Pierre Auger Observatory”, Phys. Rev. Lett. 109, 062002 (2012);

[6] The Pierre Auger Collaboration, "Measurement of the Depth of Maximum of Extensive Air Showers above $10^{18}$ eV”, Physical Review Letters, 104, 091101 (2010) (updated in Contributions to 33rd ICRC, Rio de Janeiro, Brazil, July 2013, arXiv:1307.5059);

[7] The Pierre Auger Collaboration, "The Interpretation of the Depths of Shower Maximum of Extensive Air Showers Measured by the Pierre Auger Observatory", JCAP 02 (2013) 026 (updated in Contributions to 33rd ICRC, Rio de Janeiro, Brazil, July 2013, arXiv:1307.5059);

[8] The Pierre Auger Collaboration, B. Kégl, "Measurement of the muon signal using the temporal and spectral structure of the signals in surface detectors of the Pierre Auger Observatory", in Contributions to 33rd ICRC, Rio de Janeiro, Brazil, July 2013, arXiv:1307.5059;

[9] The Pierre Auger Collaboration, I. Valiño, “A measurement of the muon number on showers using inclined events recorded at the Pierre Auger Observatory", in Contributions to 33rd ICRC, Rio de Janeiro, Brazil, July 2013, arXiv:1307.5059;

[10] The Pierre Auger Collaboration, Ioana Maris, these proceedings;

[11] The Pierre Auger Collaboration, Diego Garcia-Gamez, "Observations of the longitudinal development of extenmsive air showers with the surface detector of the Pierre Auger Observatory”, in Contributions to 33rd ICRC, Rio de Janeiro, Brazil, July 2013, arXiv:1307.5059;

[12] The Pierre Auger Collaboration, Antoine Letessier-Selvon, "Highlights from the Pierre Auger Collaboration", in the 33rd ICRC - InternationalCosmic Ray Conference, Rio de Janeiro, Brazil, July 2013, arXiv:1310.4620;

[13] The Pierre Auger Collaboration, Tanguy Pierog, "Particle Physics with the Pierre Auger Observatory", Proceedings of the 14th ICATPP Conference on Astroparticle, Particle, Space Physics and Detectors for Physics Applications, Como, Italy, September 2013. 